Title page

\title{
A Method Based on 3D Shape Analysis Towards the Design of Flexible Instruments for
}

\section{Endoscopic Maxillary Sinus Surgery}

Julie Legrand ${ }^{1,6}$, Kenan Niu ${ }^{2,6}$, Zhen Qian ${ }^{3}$, Kathleen Denis $^{1}$, Vincent Vander Poorten ${ }^{3,5}$, Laura Van Gerven $^{3,4,7}$, and Emmanuel Vander Poorten ${ }^{1,7}$

${ }^{1}$ Department of Mechanical EngineeringTechnology, KU Leuven, Leuven, Belgium

${ }^{2}$ Department of Mechanical Engineering, KU Leuven, Leuven, Belgium

${ }^{3}$ University Hospitals Leuven, Department of Otorhinolaryngology, Head Neck Surgery, Leuven, Belgium

${ }^{4}$ KU Leuven, Department of Microbiology, Immunology and transplantation, Allergy and Clinical Immunology Research Unit, Leuven, Belgium

${ }^{5}$ University Hospitals Leuven, Department of Oncology, KU Leuven, Leuven, Belgium

6 These authors contributed equally to this work and should be both considered as first author.

7 These authors contributed equally to this work and should be both considered as last author.

Correspondence: Julie Legrand. Email:julie.legrand@kuleuven.be, Kenan Niu. Email:kenan.niu@kuleuven.be

Abbreviated title: Method Towards the Design of Flexible Instruments for Endoscopic Maxillary Sinus Surgery. 


\begin{abstract}
The emergence of steerable flexible instruments have widened the uptake of minimally invasive surgical techniques. In sinus surgery, such flexible instruments could increase the access to difficult-to-reach anatomical areas. However, design-oriented metrics, essential for the development of steerable flexible instruments for maxillary sinus surgery, are lacking. This paper proposes a method to process measurements and provide the instrument designer with essential information to develop adapted flexible instruments for limited access surgery. The method was applied to maxillary sinus surgery and showed that an instrument with a diameter smaller than $2.4 \mathrm{~mm}$ could be used on more than $72.5 \%$ of the subject set. Provided that this flexible instrument can bend up to $164.4^{\circ}$, all areas within the maxillary sinus could be reached through a regular antrostomy without resorting to extra incision or tissue removal in $94.9 \%$ of the population set.
\end{abstract}

Key Terms: statistical shape model;flexible instrument;design;maxillary sinus surgery. 


\section{Introduction}

Inverted papillomas, fungus balls or carcinomas in the maxillary sinus are examples of pathologies requiring surgery. Endoscopic maxillary sinus surgery or biopsy taking requires inserting an endoscope and instruments in the maxillary sinus through the nasal cavity in order to resect or biopsy the pathological tissue. In order to be able to reach pathology inside the maxillary sinus, an antrostomy needs to be created, ${ }^{4,14}$ i.e. creating a large opening between the natural ostium of the maxillary sinus and the nasal cavity by resecting the uncinate process and removing the surrounding tissue until a window is created from the posterior border of the lacrimal canal to the posterior wall of the maxillary sinus (maximal antero-posterior diameter) and from the superior border of the inferior turbinate to the floor of the orbit (maximal height) (Figure 1). The first goal of this opening is to restore proper ventilation and drainage of the sinus. ${ }^{15}$ This medial antrostomy also offers a good window for inspection of the maxillary sinus cavity. ${ }^{14}$ The instruments that are used for maxillary sinus surgery depend on the pathology but also on the spatial location of the anomaly with respect to the antrostomy window. Currently, rigid, straight or with fixed-angled (up to $120^{\circ}$ ) instruments are being used to create this approach and perform the surgery. For visualisation, straight and rigid endoscopes with a fixed view angle of $0,30,45$ or $70^{\circ}$ are used. ${ }^{27}$ An antrostomy is not always sufficient to reach the pathology in the maxillary sinus cavity with those rigid instruments. Especially if the disease is situated on the anterior, antero-lateral or inferior wall (Figure 2a). In these specific cases, wider approaches are needed. For example, the nasolacrimal canal and the inferior turbinate may be resected. ${ }^{20}$ Alternatively, an aperture in the canine fossa may be created. ${ }^{31}$ These extra openings may lead to extra perioperative and postoperative complications. 


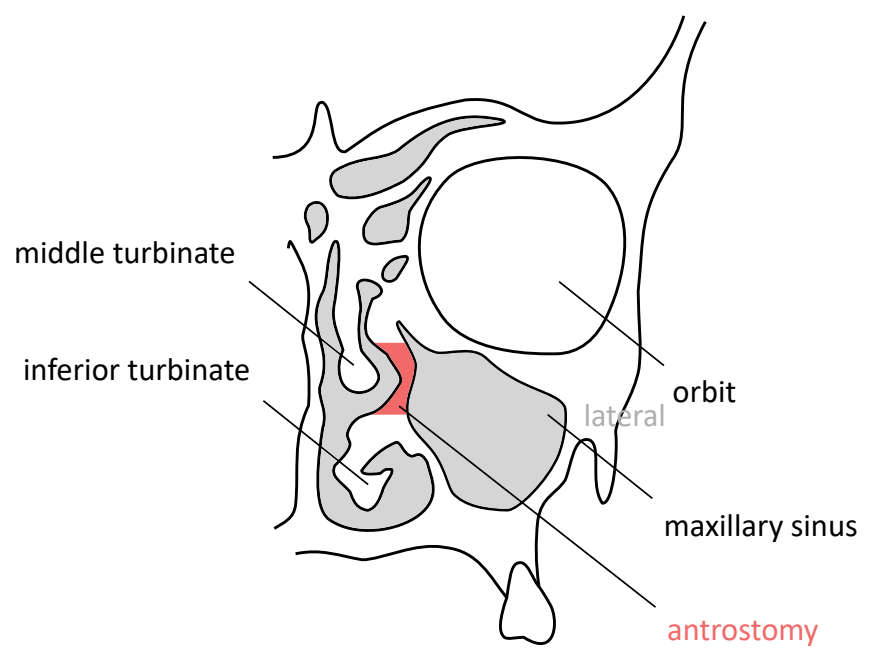

b

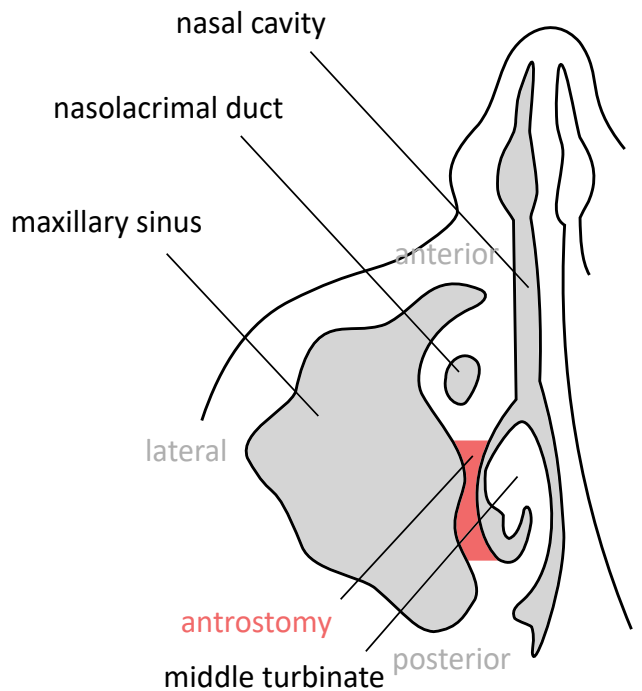

Figure 1: Maxillary sinus and nasal cavity anatomy with antrostomy procedure: resection of the tissues in red. The grey surfaces represent cavities as the white areas are bony structures; a) in the coronal plane, the resection of the tissues is performed between the inferior turbinate and the orbital wall; $b$ ) in the transverse plane, the resection is bounded by the nasolacrimal duct.

a

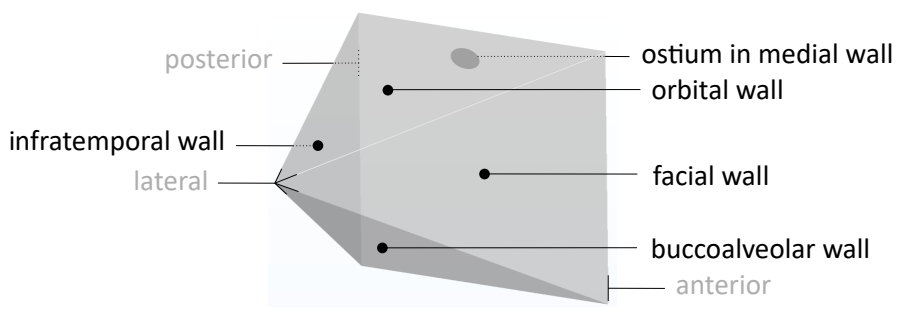

b

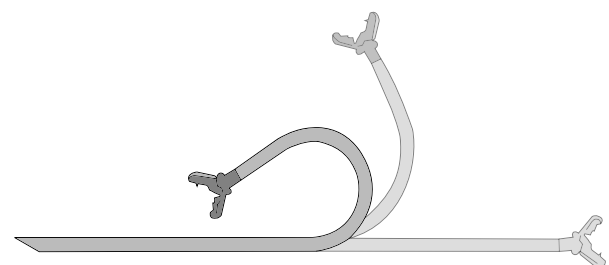

Figure 2: a. Maxillary sinus anatomy: the maxillary sinus can be seen has an horizontal pyramid composed of four walls and a base where the ostium is located; ${ }^{12} \mathrm{~b}$. Glimpse of a flexible steerable instrument.

Tichenor et al. summarized the possible advantages of flexible over rigid endoscopes to examine the maxillary sinus post-surgery. ${ }^{28}$ They emphasized the ease of use of a flexible endoscope and the ability to easily manipulate it into narrow recesses. The need for small flexible instruments and endoscopes for sinus surgery is clear. ${ }^{23}$ A conceptual example of flexible steerable instruments is presented in Figure 2b. However, anatomical data allowing the development of flexible instruments are lacking in the literature. The currently available data relating to sinuses were either collected 
manually on cadavers, ${ }^{9,1,29}$ manually on 2D computed tomography (CT) scans ${ }^{17,7,3,21,26,24}$ or manually from a reconstructed 3D surface obtained by segmentation of CT scans. ${ }^{8,13}$ The measurements taken on cadavers as well as the data collected from 2D CT scans are non-specific. Only general dimensions such as the maxillary sinus length, height, width, volume or height of septa were measured. The measurements manually taken from a reconstructed $3 \mathrm{D}$ surface are general and mainly used as an automatic tool for gender determination or analysis of the ageing process on the maxillary sinus aeration. This type of data is not sufficient and detailed enough to be used as technical specifications for the design of flexible instruments. Moreover, the data only focuses on the maxillary sinus itself and does not give any information on the nasal cavity or its relative position with respect to the maxillary sinus which is important to reach the site of surgery without damaging the septum and turbinates.

This paper proposes a method to collect design-oriented metrics on the nasal cavity and the maxillary sinus. The method can provide the instrument designer with enough information to develop an adapted flexible instrument for maxillary sinus procedures. This method consists out of three phases. First, cranial CT scans are collected. These CT scans are segmented in order to obtain for each scan, two maxillary sinus and two half-nasal cavity shapes. Second, a statistical shape model (SSM) computes the mean shape and the shape variations of the maxillary sinuses and nasal cavities. SSMs are widely used in medical image processing, such as image segmentation, ${ }^{16}$ image registration ${ }^{2}$ and image reconstruction. ${ }^{10}$ SSMs allows to quantify geometrical variations and features from various shapes and anatomical structures. Knowledge about the expected 
variations among a group of subjects can provide key information for, amongst others, the design of artificial implants ${ }^{30}$ and prostheses. ${ }^{22}$ Following a similar reasoning, the use of an SSM of the nasal cavity and maxillary sinus would allow the analysis of the geometrical variations of the targeted anatomy and the investigation of the impact of this variability on the instrument shape to be designed. Till date the studies on SSMs of paranasal sinuses essentially focused on automatic image segmentation. ${ }^{16,25}$ There is up to now, to the best of the author's knowledge, no study that uses an SSM to guide the design procedure for steerable flexible surgical instruments. Third, design-oriented metrics are selected based on the SSM. A large number of dimensions need to be identified to devise an instrument that can be deployed for a large majority of maxillary sinus disorders. The proposed workflow is generic and can be applied to various kinds of surgical procedures in order to facilitate the design process and clarify the design requirements of surgical instruments.

\section{Materials and Methods}

\subsection{CT image data}

CT scans were collected from 20 healthy subjects (10 males and 10 females, $43 \pm 15.14$ years) that had no disease in the nasal cavity and paranasal sinuses. This is indicated as the first step in the processing workflow that is depicted in Figure 3. One subject was found to have a serious form of septum deviation. This subject was intentionally kept in the data set since a septum deviation is

not a rare anomaly. The obtained data set therefore represents a relatively realistic sample of the population. The CT scans were acquired using a Cone Beam CT (VGI EVO, NewTom, Verona, 
Italy) with a $0.25 \mathrm{~mm} \times 0.25 \mathrm{~mm}$ pixel resolution in plane and a slice thickness between 0.25 and $1 \mathrm{~mm}$. Ethical approval for using this data was obtained from UZ Leuven Ethics Committee (Gasthuisberg, Leuven, Belgium, file number S63078). The collected CT images were manually segmented using Mimics version 20.0 (Materialise, Leuven, Belgium) (step 2 in Figure 3) to get the 3D surface meshes of the nasal cavity and the maxillary sinus. In addition, the antrostomy window was manually created during image segmentation to connect the nasal cavity and maxillary sinus (red area in Figure 1) mimicking the surgical path that is followed during today's maxillary sinus surgery. The window was segmented by an engineer under supervision of an experienced ear, nose, throat, head and neck (ENT HN) surgeon. The left and right sinuses were segmented separately with their respective nasal cavity part. The segmented surface meshes were exported as STL files using Mimics. This resulted in 20 left-side and 20 right-side surface meshes of the maxillary sinus and the nasal cavity (step 3 in Figure 3).

\subsection{SSM of the nasal cavity and maxillary sinus}

A subject's left and right sinuses are not exactly identical. The surface meshes of the left sinuses were thus mirrored to the right ones which increased the shape variations of the obtained surface meshes. 40 different surface meshes of right sinuses with half of the nasal cavity were obtained in total (step 3 in Figure 3). In order to build an SSM using principal component analysis (PCA), ${ }^{5}$ point correspondences need to be established among all surface meshes. This means that for a given point located on one surface mesh, a corresponding point, located on a similar anatomical area, must be found on each of the 39 other surface meshes. To establish the correspondences an arbitrary 
(1)
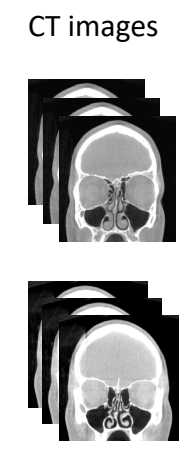

:

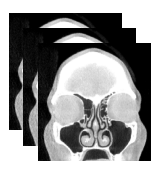

(2)

Manual segmentation
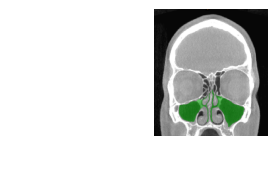

(3)

Surface meshes

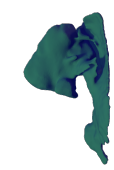

(4)

Elastic

registration

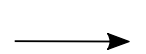

(5)

Replicated meshes
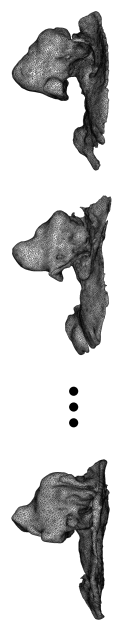

(7)

\section{SSM}

(6)

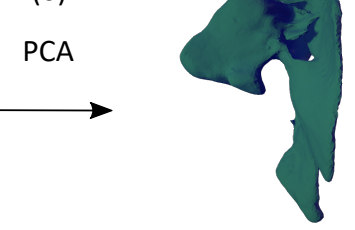

Figure 3: Workflow for building a statistical shape model of the nasal cavity and the maxillary sinus; (1) cranial CT scan collection; (2) manual segmentation of the maxillary sinuses and nasal cavities; (3) creation of 3D surface meshes for each right sinus and half-right nasal cavity, and mirroring of the left sinus and half-left nasal cavity; (4) elastic registration of the 3D meshes; (5) acquisition of the point correspondence meshes; (6) application of principal component analysis; (7) acquisition of the SSM of the right maxillary sinus and half of the nasal cavity.

mesh was selected as the reference mesh. An elastic registration was then performed to register the reference mesh to the rest of the meshes (step 4 in Figure 3). ${ }^{6}$ The replicated meshes, also named point correspondence meshes (PCMs), therefore contain the same number of corresponding points as the reference mesh. Subsequently, the SSM can be built by applying PCA on the vertices of all PCMs together with the reference mesh (step 5-7 in Figure 3). It results in a mean shape $\bar{U}$ and a set of modes of variations representing the deviations of the training shapes with respect to the mean shape. These modes of variations are called shape modes or eigenvectors. A shape can therefore be represented as a linear combination of the shape modes together with the mean shape: ${ }^{5}$

$$
\mathrm{U}=\overline{\mathrm{U}}+\mathbf{b} \phi
$$


where $\bar{U}$ is the mean shape vector, $\phi=\left[\phi_{1}, \phi_{2}, \ldots, \phi_{t}\right]$ is the matrix of the first $t$ eigenvectors and $\mathbf{b}=\left[b_{1}, b_{2}, \ldots, b_{t}\right]$ is the vector that describes the weights of each eigenvector. Equation (1) allows to generate a new shape example $U$ by changing the parameters $b_{t}$ within proper limits. The variance of $b_{t}$ over the training set equals to $\lambda_{t}$. $\lambda_{t}$ represents the $t^{t h}$ eigenvalue corresponding the $t^{t h}$ eigenvector $\phi_{t}$. The range of $b_{t}$ is commonly limited to $-3 \sqrt{\lambda_{t}} \leq b_{t} \leq 3 \sqrt{\lambda_{t}}$, since most of the deviations lie within three standard deviations of the mean.

\subsection{Geometrical parameters extraction}

In this study, the mean shape and the shape variations obtained by the SSM are used to extract a series of useful design-oriented metrics that can guide the design procedure of new steerable flexible surgical instruments. In this context, one can identify the dimensions of the maxillary sinus and nasal cavity morphological structure that constrain the available work envelope of a flexible instrument to be designed. Those dimensions can be used to determine the required length, outer diameter, bending angle, etc. of the flexible instrument. Given that the SSM also provides the maxillary sinus and nasal cavity shape variations, it is possible to study the effects of the morphological variations directly on the design-oriented metrics.

In order to identify those design-oriented metrics, it is important to analyse the path the instrument needs to follow to reach the maxillary sinus walls (Figure 2a). During an endoscopic maxillary sinus procedure, diverse rigid instruments are inserted through the nose in order to create the antrostomy. This antrostomy is systematically performed since it helps to restore proper ventilation 
and drainage of the sinuses and increases the chances of full recovery after surgery. Once this access window is created, endoscopes and graspers are inserted through the nose going through the antrostomy until the maxillary sinus. Up to now, those instruments are straight and rigid. A steerable flexible instrument that would access the maxillary sinus without creating any additional incision other than the antrostomy would be beneficial for shorter surgical procedures and shorter patient's recovery time. The shape of this flexible instrument could be adjusted to accommodate and enter the maxillary sinus through the antrostomy, and visualize and/or reach the walls of the maxillary sinus. The expected surgical path for such instrument can be divided in two steps. First, the nasal cavity insertion, where the instrument is introduced into the nostril, driven into the nasal cavity to reach the antrostomy window. Second, the maxillary sinus exploration, where the flexible instrument has entered the maxillary sinus cavity and is manipulated to reach the anomalies located on the maxillary sinus walls. Based on both steps, several specific landmarks where chosen on the obtained shapes by engineers in order to characterize the overall dimensions of the flexible instrument (Figure 4). These landmarks either determine the general path the instrument needs to take from the entry point to reach maxillary sinus walls or characterize the boundaries defined by the maxillary sinus and nasal cavity anatomy. Typically, the entry point and the middle point of the antrostomy window are used to define the instrument path. The height, width and thickness of the antrostomy window, the center point of the maxillary sinus and the radius of the sphere including the maxillary sinus define the boundaries. The landmarks taken on the maxillary sinus and nasal cavity are summarized in Table 1 . 
The surgical entry point, i.e. the middle of the nostril (landmark $a$ in Figure 4), was manually selected on the reconstructed SSM. The antrostomy window characteristics, namely its length $d$ , width $g$, and thickness $e$, were measured after manually selecting multiple points on the outer surface of the antrostomy window (points $P_{a w}$ in Figure $4 \mathrm{c}$ ). Then, a singular value decomposition was applied to find the three eigenvectors corresponding to the three largest eigenvalues of the covariance matrix. Finally, these points were transformed in a new coordinate system $\left(X_{a w, s v d}\right.$, $\left.Y_{a w, s v d}, Z_{a w, s v d}\right)$ in which the $X Y Z$ axes align with the found eigenvectors. This results in a new point cloud $P_{a w, s v d}$ (Figure 4c). The $X\left(X_{a w, s v d}\right), Y\left(Y_{a w, s v d}\right)$ and $Z$ axis $\left(Z_{a w, s v d}\right)$ of the new coordinate system align with the directions of the length $d$, width $g$ and thickness $e$ of the antrostomy window respectively. The values of $d, g$ and $e$ are obtained from below Eqs. (2), (3) and (4) respectively. The central point of the antrostomy window $b$ is calculated by averaging $P_{a w}$.

$$
\begin{gathered}
d=\max \left(X_{a w, s v d}\right)-\min \left(X_{a w, s v d}\right) \\
g=\max \left(Y_{a w, s v d}\right)-\min \left(Y_{a w, s v d}\right) \\
e=\max \left(Z_{a w, s v d}\right)-\min \left(Z_{a w, s v d}\right)
\end{gathered}
$$

The landmarks $h$ and $i$, namely the most lateral and anterior point of the maxillary sinus respectively, were manually selected. The maxillary sinus was virtually detached from the nasal cavity, and a sphere, passing by the landmarks $h$ and $i$ was fitted by least square optimization onto the mesh of the maxillary sinus alone. The radius of this fitting sphere $f$ and its centre $c$ were then computed.

The points reported in Table 1 were selected from the surface of the SSM, therefore, the various 
a

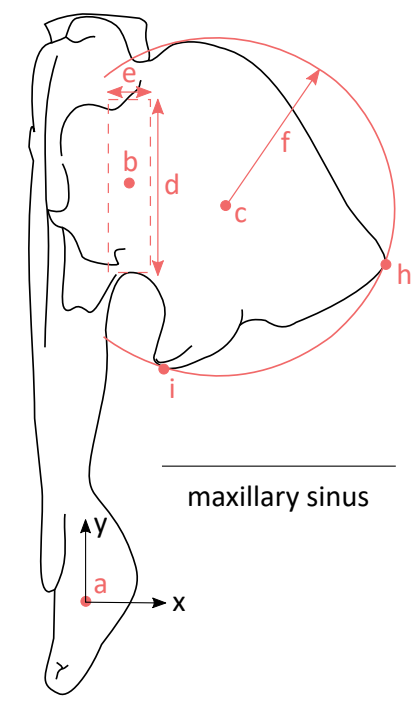

nasal cavity

C

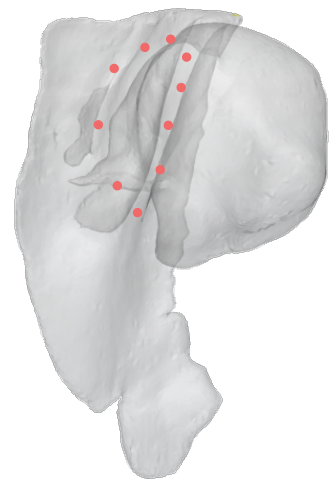

(1) b
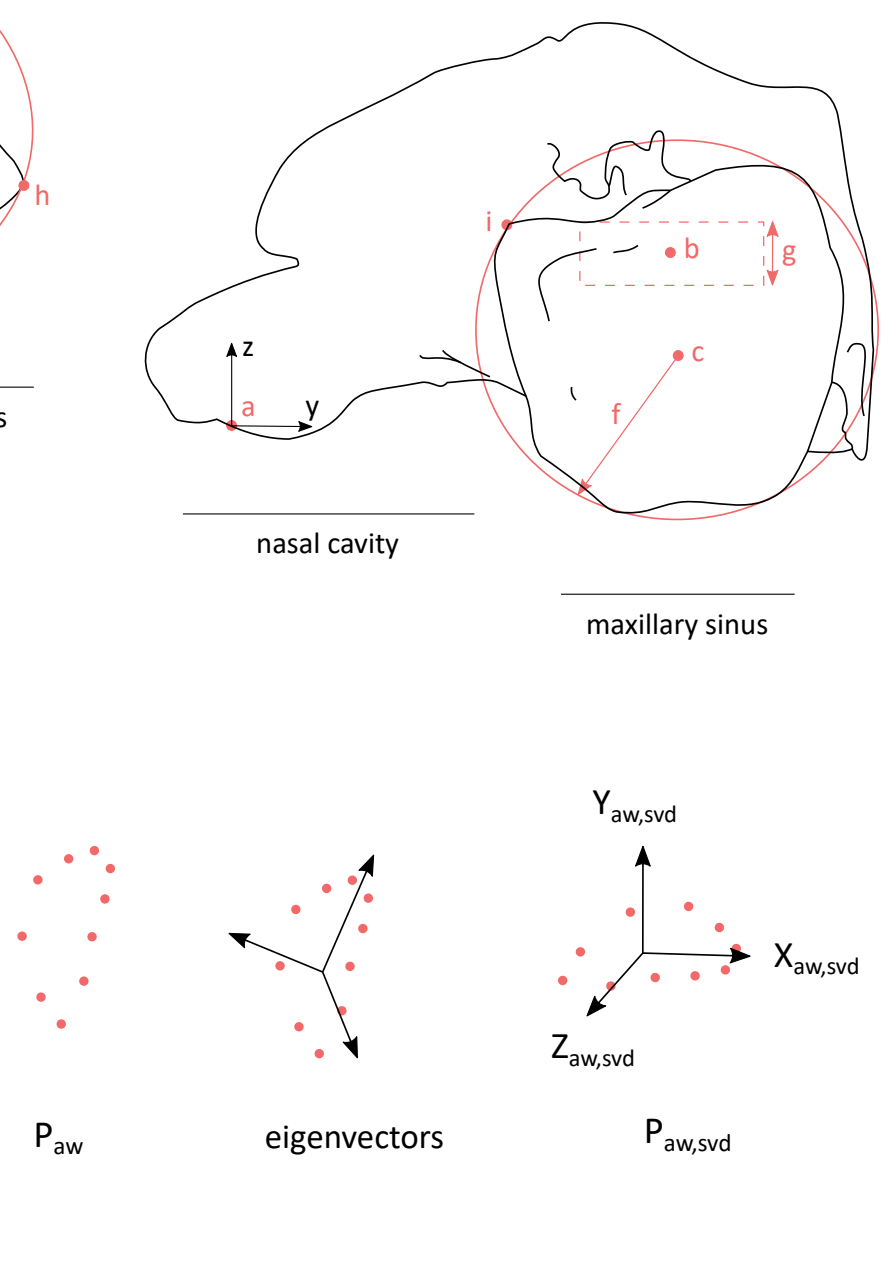

(2)

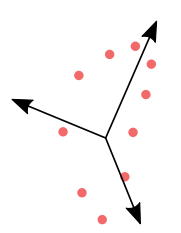

eigenvectors

(3)

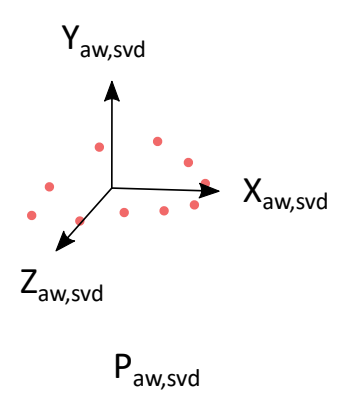

(4)

Figure 4: Definition of landmarks and parameters on the nasal cavity and the maxillary sinus. a) transverse plane; b) sagittal plane; c) diagram deriving the antrostomy window geometry: (1) manual selection of points on the outer surface of the antrostomy window; (2) the obtained points are in the same coordinate frame as the SSM; (3) three eigenvectors that correspond to the first three largest eigenvalues are calculated; (4) transformation of the points in a new coordinate frame $\left(X_{a w, s v d}, Y_{a w, s v d}, Z_{a w, s v d}\right)$ where the length, width and thickness of the antrostomy window can be measured.

shape modes of the SSM that change from $-3 \sqrt{\lambda_{t}}$ to $3 \sqrt{\lambda_{t}}$ will lead to an accordingly representative distribution of the strategic key points of Table 1 . Note that $3 \sqrt{\lambda_{t}}$ represents 3 times the standard deviation. Considering all the shape variations between $-3 \sqrt{\lambda_{t}}$ and $3 \sqrt{\lambda_{t}}$ therefore takes into account $99.7 \%$ of all the variations present in the data set for a given mode. The position 
and values of the landmarks as well as their mean and variations are reported in Section 3 for the different shape modes.

Table 1: Definition of the landmarks.

\begin{tabular}{lccc}
\hline Type & Landmarks & Physical meaning & Acquisition mode \\
\hline 3D point & $\mathrm{a}$ & nostril middle point & manual \\
3D point & $\mathrm{b}$ & antrostomy window center point & automatic \\
3D point & $\mathrm{c}$ & maxillary sinus center point & automatic \\
length & $\mathrm{d}$ & antrostomy window length & automatic \\
thickness & $\mathrm{e}$ & antrostomy window thickness & automatic \\
radius & $\mathrm{f}$ & radius of the maxillary sinus fitting sphere & automatic \\
width & $\mathrm{g}$ & antrostomy window width & automatic \\
3D point & $\mathrm{h}$ & most lateral point of the maxillary sinus & manual \\
3D point & $\mathrm{i}$ & most anterior point of the maxillary sinus & manual \\
\hline
\end{tabular}

The landmarks are identified on the maxillary sinus and nasal cavity in accordance with Figure 4.

\subsection{Nasal cavity width analysis}

The width of the nasal cavity constrains the outer diameter of the flexible instrument to be designed. Therefore, a width analysis on the SSM of the nasal cavity is proposed. In order to estimate the nasal cavity width, the centerline of the nasal cavity and the maxillary sinus from the nostril middle point $a$ to the most lateral point of the maxillary sinus $h$ is first obtained. To do so, a skeletonization algorithm provided by the VMTK (1.4.0) library4 was used (Figure 5a). For each shape mode, the mean centerline and its $\pm 3 \sqrt{\lambda_{t}}$ variations were extracted. Note that the calculated centerline forms only one possibility of an admissible surgical path that can be followed by the flexible instrument. A more in-depth study of all possible surgical paths is beyond the scope of this paper. However, the centerline can be considered as a practicable path to steer the flexible instrument along. The 


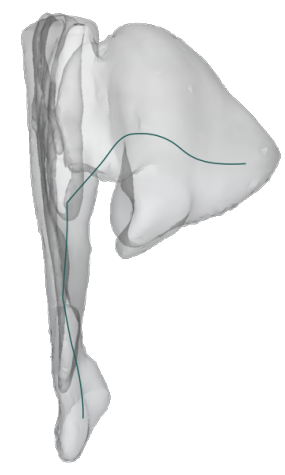

(a)

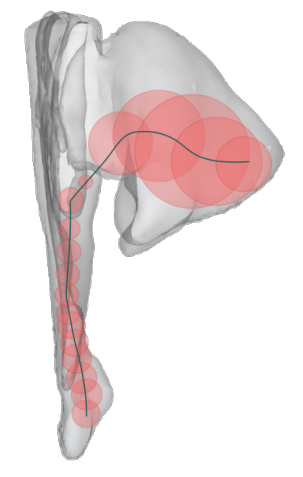

(b)

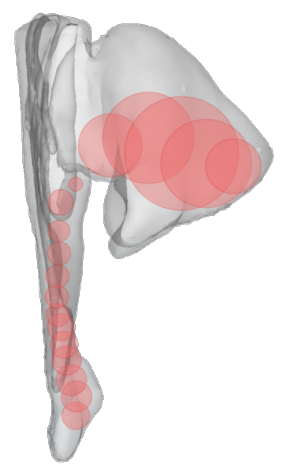

(c)

Figure 5: Method used to extract the nasal cavity width: (a) acquisition of the centerline using skeletonization; (b) creation of the maximal inscribed spheres having the centerline as center; (c) resulting maximal inscribed spheres.

$3 \mathrm{D}$ coordinates of the centerline of the mean shape and the $\pm 3 \sqrt{\lambda_{t}}$ variations of the first mode are reported in result Section 3.

The distance between the points located on the centerline and their corresponding closest point on the anatomical surface is then computed. This distance represents the radius of the maximal allowable inscribed sphere for a point on the centerline. The evolution of maximal inscribed spheres along the centerline is then acquired (Figure 5b). The diameter of these spheres represents the width of the nasal cavity and maxillary sinus which will constrain the flexible instrument external diameter. The spheres are smaller inside the nasal cavity than in the maxillary sinus and are thus more restrictive regarding the instrument outer diameter. The results of the nasal cavity width analysis are reported in the result Section 3.

\section{Results}

The SSM was built from 40 surface meshes of the nasal cavity and the maxillary sinus, resulting in one mean shape and 39 shape modes. Note that no distinction was made between the male and 
female shapes. This is because the flexible instrument to be designed is intended to be used for both genders. The landmark values therefore include all the collected surface meshes regardless of the subject's gender.

From analysing the variance of each individual's eigenvalue of the built SSM (Figure 6a), it was found that the first mode explains $26.13 \%$ of the total variation in the set. The second and third mode explains $8.9 \%$ and $7.1 \%$ of the variations respectively. Together, the first 5, 10 and 27 modes explains $54 \%, 71.6 \%$ and $95.2 \%$ of the variations respectively. This section will therefore focus on the results of the first 27 modes, since the SSM is able to represent $95.2 \%$ of the total set variations.

\subsection{SSM shape modes}

In order to investigate the effects of the different shape modes on the landmarks values, it is possible to generate each shape mode separately. Figure $6 \mathrm{~b}$ illustrates the shape variations between $-3 \sqrt{\lambda_{t}}$ and $3 \sqrt{\lambda_{t}}$ as well as the mean shape for the first two shape modes. From Figure $6 \mathrm{~b}$ one can observe that the main geometrical change in the first shape mode is the volume of the maxillary sinus and nasal cavity. The second shape mode mainly includes morphological changes. More specifically, the orbital and infratemporal wall of the maxillary sinus becomes larger from $-3 \sqrt{\lambda_{t}}$ to $3 \sqrt{\lambda_{t}}$ whereas the height of the maxillary sinus become smaller.

\subsection{Landmarks}

Based on the designed SSM, the values of the different landmarks, identified in Figure, 4 could be acquired. Table 2 gathers the mean and the largest variations of each landmark. In this table, the 
a

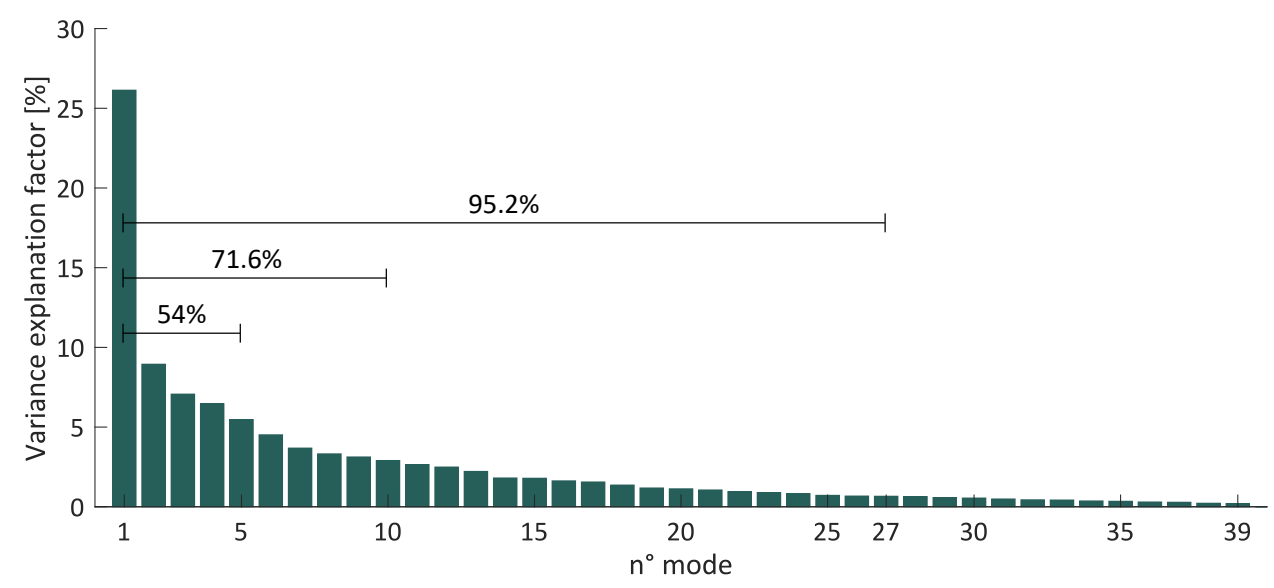

b

Mode 1

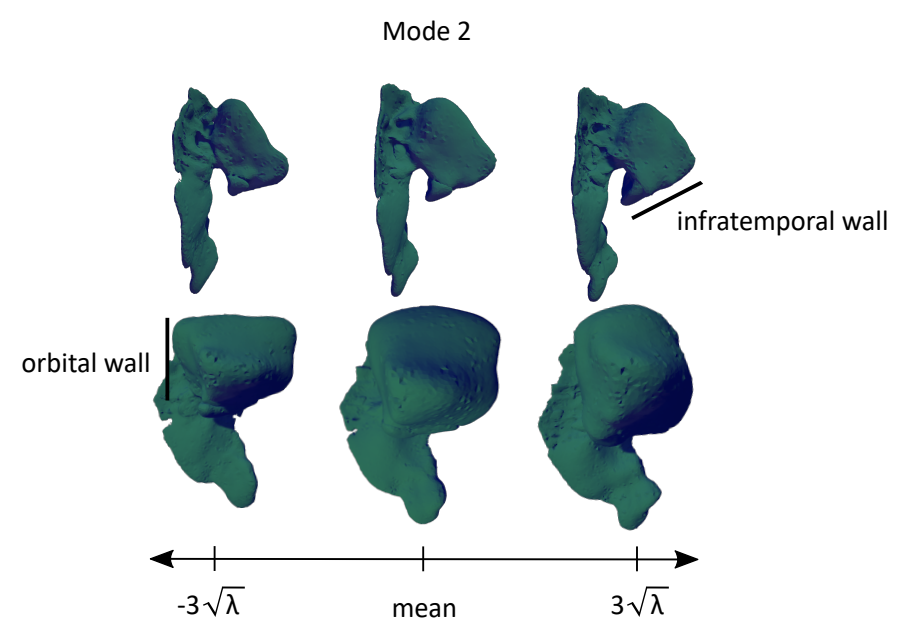

Figure 6: a) Variance of each individual eigenvalue in percentage of the built SSM. The first shape mode represents $26.13 \%$ of the total set variations. The first 5, 10 and 27 modes explain together 54\%, 71.6\% and $95.2 \%$ of the total set variations respectively; b) visualization of the mean shape for the first two shape modes from $-3 \sqrt{\lambda_{t}}$ to $3 \sqrt{\lambda_{t}}$ of the nasal cavity and the maxillary sinus. The first mode shows changes in size, whereas the second mode shows morphological changes.

mode column indicates the mode for which the largest variation was recorded. For practically all the landmarks the largest variations were recorded within the first 5 modes. The detailed landmark measurements for the 27 first modes are reported in Appendix A, in Figures 9 - 14. For the landmarks of type $3 D$ point, the 3D coordinates are given with respect to the entry point $a$, the origin of the frame. The mean position and variations $-3 \sqrt{\lambda_{t}}$ and $3 \sqrt{\lambda_{t}}$ of the entry point $a$ are therefore set to $(0,0,0)$ for each mode. From table 2, one can observe that $c_{x}$ and $h_{x}$, the x-coordinate of $c$ and $h$, 
and $i_{z}$, the $\mathrm{z}$-coordinate of $i$, show the largest variations $(> \pm 5 \mathrm{~mm})$. Taking the variations between $-3 \sqrt{\lambda_{t}}$ and $3 \sqrt{\lambda_{t}}$ into account for the first 27 modes allows to represent $95 \%$ of all the variations present in the data set $\left(\frac{99.7 \% \times 95.2 \%}{100}\right)$.

Table 2: Mean and maximal variations of the extracted landmarks.

\begin{tabular}{lcclcc}
\hline landmarks & mean $\left( \pm \mathbf{3} \sqrt{\lambda_{t}}\right)$ & mode & landmarks & mean $\left( \pm \mathbf{3} \sqrt{\lambda_{t}}\right)$ & mode \\
\hline$b_{x}$ & $2.18( \pm 1.74)$ & 5 & $f$ & $16.62\left(\left(_{-3.45}^{+3.73}\right)\right.$ & 1 \\
$b_{y}$ & $55.06( \pm 3.00)$ & 5 & $g$ & $14.31\left(\left(_{-3.4}^{+5.95}\right)\right.$ & 2 \\
$b_{z}$ & $10.45( \pm 3.85)$ & 2 & $h_{x}$ & $31.59( \pm 5.09)$ & 1 \\
$c_{x}$ & $16.90( \pm 5.56)$ & 4 & $h_{y}$ & $51.4( \pm 3.04)$ & 3 \\
$c_{y}$ & $51.84( \pm 3.18)$ & 3 & $h_{z}$ & $6.46( \pm 4.33)$ & 2 \\
$c_{z}$ & $3.62( \pm 3.20)$ & 2 & $i_{x}$ & $9.53( \pm 2.50)$ & 4 \\
$d$ & $28.78\left(\begin{array}{c}+4.21 \\
-3.94\end{array}\right)$ & 1 & $i_{y}$ & $31.76( \pm 4.47)$ & 5 \\
$e$ & $2.79\left(\begin{array}{l}+1.26 \\
-0.88\end{array}\right)$ & $1 ; 13$ & $i_{z}$ & $16.85( \pm 7.06)$ & 1 \\
\hline
\end{tabular}

Mean and maximal variations of the extracted landmarks $b, c, d, e, f, g, h$ and $i$ values in [mm]. The mode column indicates the mode for which the maximal variations were recorded.

\subsection{Nasal cavity width}

The nasal cavity widths were obtained from the diameter calculation of the maximal inscribed spheres located along the centerlines (see Section 2). Figure 7a shows the mean and the minimum and maximum values of the $\pm 3 \sqrt{\lambda_{t}}$ variations over the 27 first modes of the obtained centerline from point $a$ until the center of the antrostomy window (point $b$ ). From this figure, one can observe that the variations of the centerline in the $y$-z plane are large (about $\pm 5 \mathrm{~mm}$ from the mean). This shows that the maxillary sinus can be situated higher or lower in the y-z plane depending from the subject whereas its position in the $x-y$ plane shows less variability. The flexible instrument to be 
designed should be capable to navigate within the limits of the grey area depicted in Figure 7a.

Figure $7 \mathrm{~b}$ depicts the calculated nasal cavity widths along the y axis from the origin point, i.e. the nostril middle point, until the center of the antrostomy window (point b). It can be observed that the mean nasal cavity width decreases moving away from the nostril. In this figure, the surface $a$ represents the modes and variations for which the nasal cavity width is larger than $3 \mathrm{~mm}$. These are modes $3,7,9,11,15,16,18,21,23,24$, and 27 for $+3 \sqrt{\lambda_{t}}$, and modes $6,8,10,11,12,13,15,20$, 21,22 , and 25 for $-3 \sqrt{\lambda_{t}}$. Together, those modes and variations represent $22.3 \%$ of the subject's set. This percentage was calculated using the following formula:

$$
\mathrm{p}=\frac{\left(\sum \mathrm{VEF}_{\text {mode }}^{\mathrm{c}} \times 99.7\right)+\left(\sum \mathrm{VEF}_{\text {mode }}^{\mathrm{i}} \times \frac{99.7}{2}\right)}{100}
$$

were $p$ represents the obtained percentage of the subject sets, $V E F_{\text {mode }}^{c}$, the variance explanation factor (in percent) (see Figure 6a) for the modes for which both variations $\pm 3 \sqrt{\lambda_{t}}$ are higher than an arbitrary nasal cavity width limit, $V E F_{\text {mode }}^{i}$, the variance explanation factor for the modes for which either the variation $+3 \sqrt{\lambda_{t}}$ or $-3 \sqrt{\lambda_{t}}$ is higher than the arbitrary limit. 99.7 represents the percentage of the data that are within $\pm 3 \sqrt{\lambda_{t}}$, considering a normal distribution of the data. Eq (5) somewhat underestimates the percentage of the subject set. When one of both variations $\pm 3 \sqrt{\lambda_{t}}$ is exceeding the width arbitrary limit, the entire half side of the Gaussian curve is discarded, letting only $\frac{99.7}{2} \%$ as percentage for the considered mode. This means that all the variations within $\pm 3 \sqrt{\lambda_{t}}$ were not tested. 
a
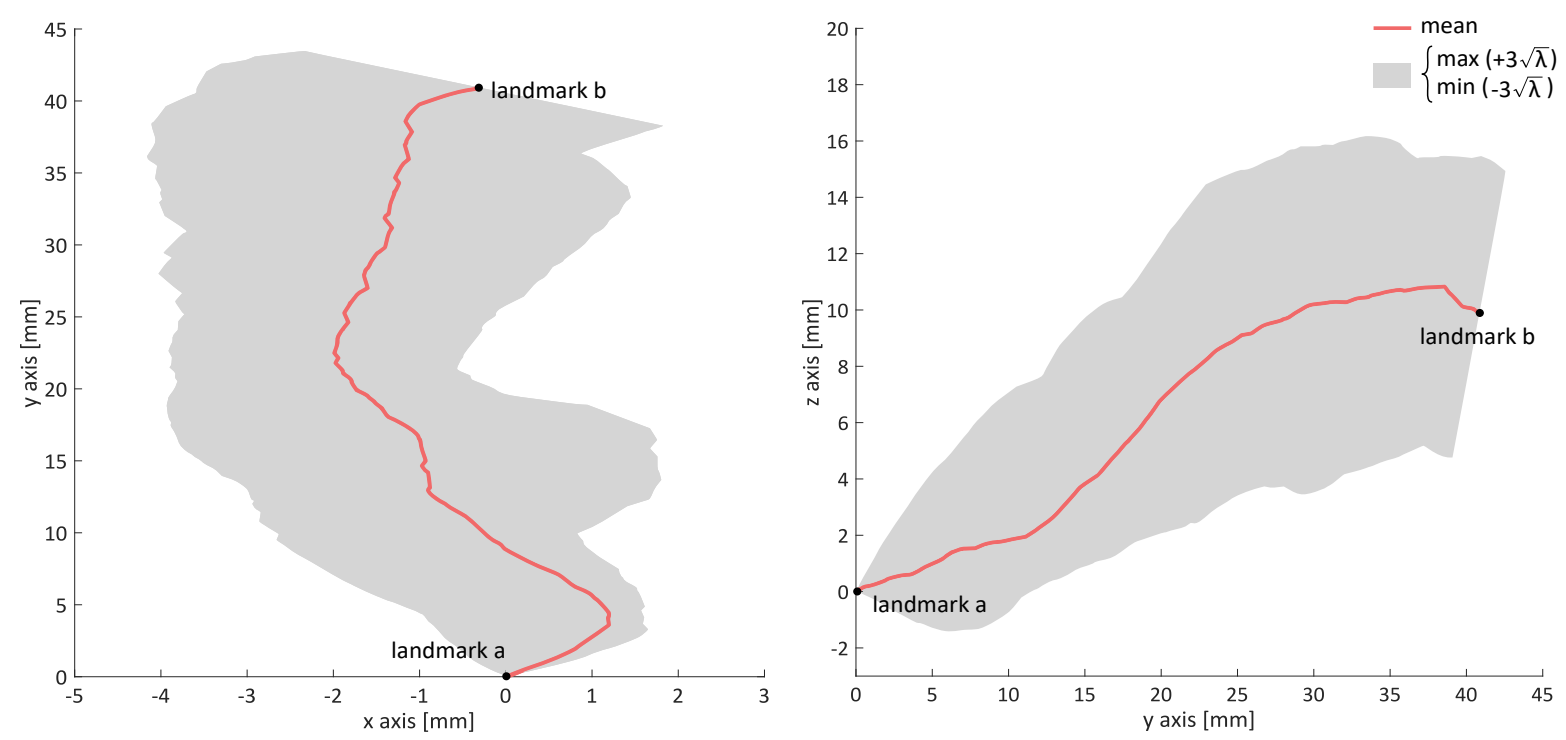

b

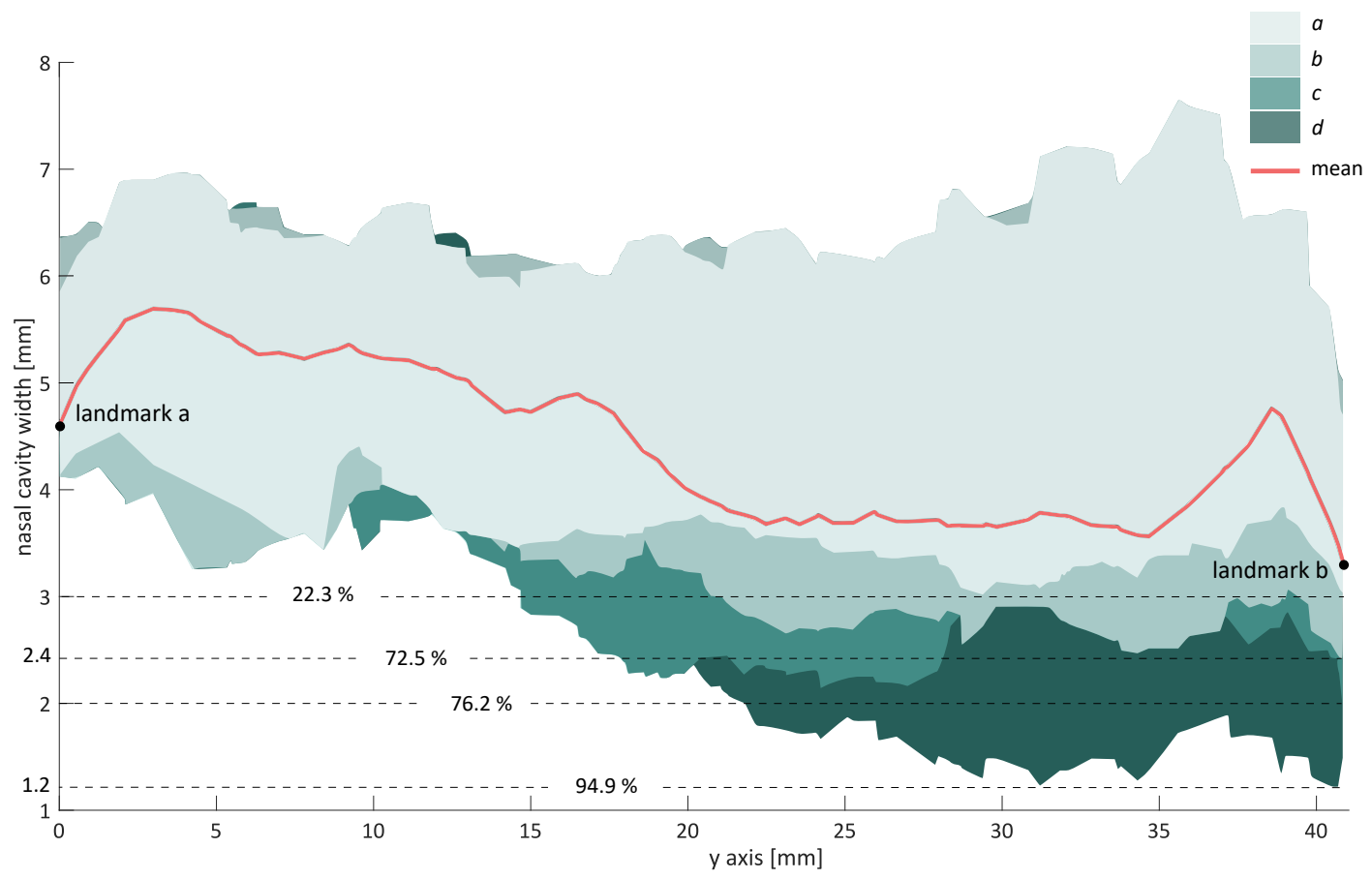

Figure 7: a) coordinates of the centerline, mean and maximum and minimum of the $\pm 3 \sqrt{\lambda_{t}}$ variations for the first 27 modes from point $a$ until the center of the antrostomy window (point $b$ ); b) nasal cavity width along the $y$-axis from the nostril middle point to the center point of the antrostomy window; $a$. Modes and variations for which the nasal cavity width is larger than $3 \mathrm{~mm} ; b$. Modes and variations for which the nasal cavity width is larger than $2.4 \mathrm{~mm}$; . Modes and variations for which the nasal cavity width is larger than 2 $\mathrm{mm} ; d$. All 27 modes and $\pm 3 \sqrt{\lambda_{t}}$ variations. The minimum nasal cavity width is $1.2 \mathrm{~mm}$ considering $94.9 \%$ of the subjects set. 
The surface $b$ from Figure $7 \mathrm{~b}$ represents the modes and variations for which the nasal cavity width is larger than $2.4 \mathrm{~mm}$. These are all the modes except modes 4, 5, 6, 8, 10 and 17 for $+3 \sqrt{\lambda_{t}}$ and all the modes except modes $2,3,9$, and 16 for $-3 \sqrt{\lambda_{t}}$. Together, those modes and variations represent $72.5 \%$ of the subject's set. The surface $c$ represents the modes and variations for which the width is larger than $2 \mathrm{~mm}$. These are all the modes besides modes $4,5,6$, and 8 for $+3 \sqrt{\lambda_{t}}$, and all the modes besides 2,3 , and 16 for $-3 \sqrt{\lambda_{t}}$. Together, those modes and variations represent $76.5 \%$ of the subjects set. Finally, the surface $d$ regroups all the modes and variations, which represent $94.9 \%$ of the subject's set $\left(\frac{99.7 \% \times 95.2 \%}{100}\right)$. The minimum nasal cavity width was measured to be $1.2 \mathrm{~mm}$ when taking the 27 modes and variations $\pm 3 \sqrt{\lambda_{t}}$ into account.

If one wants to design a flexible instrument that can be used on $94.9 \%$ of the subject's set, the instrument external diameter has to be $1.2 \mathrm{~mm}$ or less in order to avoid the damage of any extra tissue. Building such a small flexible instrument can, however, be very challenging. A flexible instrument with an external diameter of $2 \mathrm{~mm}$ could be used on $76.2 \%$ of the subject set without extra tissue dissection. A $2.4 \mathrm{~mm}$ external diameter instrument could be used on $72.5 \%$ of the subject set, while a $3 \mathrm{~mm}$ one would only be usable on $22.3 \%$ of the set without resorting to extra tissue dissection.

\section{Discussion}

In endoscopic maxillary sinus surgery it is found difficult to reach targets inside the maxillary sinus with rigid instruments without performing additional incisions. The accessible area inside the 
maxillary sinus could however be extended without additional incisions if a steerable, flexible instrument would be available. Designing such a flexible instrument is non-trivial given the large variability amongst the subjects sinus anatomy. A large set of design-oriented data is therefore needed. However, it is not straightforward to draw conclusions from an extensive data set without following a structured approach. Therefore, a SSM based method was proposed that models the morphological and shape changes of the nasal cavity and the maxillary sinus. Similar work has been proposed for optimizing orthopedic implants design by using SSM. ${ }^{18}$ More specifically, SSM and a level-set evolution algorithm were used to search for an optimal fitting between the designed implants and fractured bones. The proposed SSM method was used here to derive design-oriented metrics for designing new flexible instruments for endoscopic maxillary sinus surgery. By doing so, the obtained results allow to provide the designer with a structured approach to deal with the complexity of variable anatomy and derive instrument-related parameters, such as its external diameter, bending angle or length. Based on Figure $7 \mathrm{~b}$ it is possible to deduce the external diameter of the steerable flexible instrument based on the targeted proportion of the subjects set. A diameter smaller than $2.4 \mathrm{~mm}$ can take more than $72.5 \%$ of the subject set into account. This result highlight the fact that progress still need to be made in the development of thin flexible instruments for sinus surgery since the latest instruments presented in the literature have a diameter of $4 \mathrm{~mm} .{ }^{32,11}$ Note that ENT surgeons can use more than one instrument at a time inside the maxillary sinus. The instrument to be designed can therefore be equipped with more than one tool, as the flexible instrument developed by Legrand et al. for fetal surgery. ${ }^{19}$ It is further possible to deduce the maximum deflection angle that the flexible instrument needs bend to be able to enter the maxillary sinus. Indeed, by calculating the angle between both vectors $\overrightarrow{a b}$ and $\overrightarrow{b i}$ based on the data presented 
in Appendix A, one can obtain the required bending angle. Figure 8 depicts the instrument bending angle for the first 27 modes. From this figure, it can be deduced that a maximum bending angle of $164.5^{\circ}$ suffices in order to be able to access the inside of the maxillary sinus in $94.9 \%$ of the population set without resorting to any extra incision.

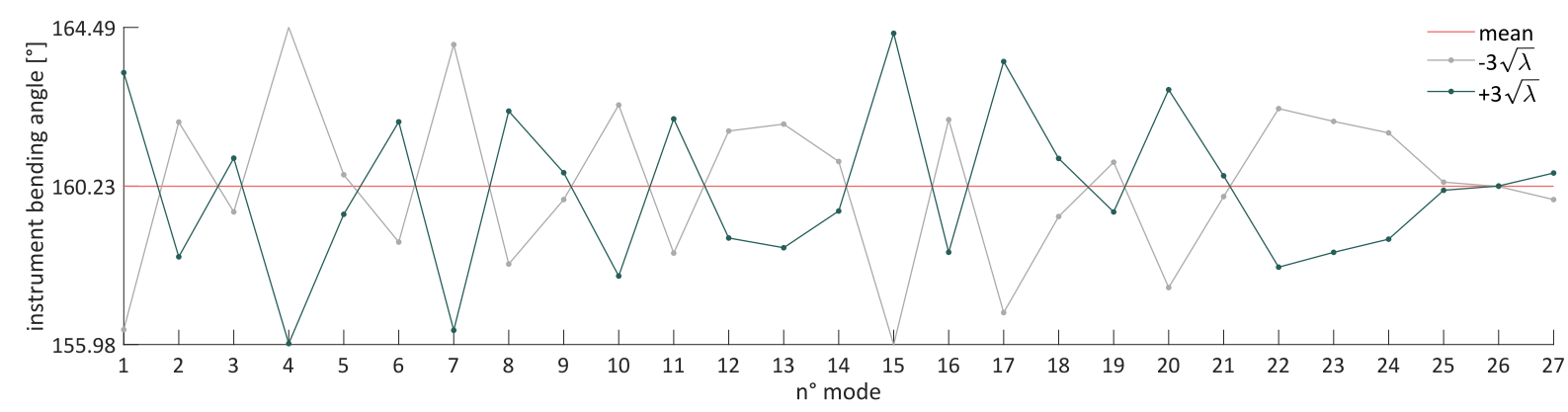

Figure 8: Angle (in degrees) between both vectors $\overrightarrow{a b}$ and $\overrightarrow{b i}$ in the x-y plane (see Figure 4a) representing the required bending angle of the flexible instrument to be able to enter the maxillary sinus.

This analysis of the maximum bending angle is a second example that shows how the data obtained in Section 3 can be used to deduce interesting design-oriented metrics. Using the data given in Appendix A, the designer can fix a number of degree of freedom for his/her instrument depending on its function. In function of the chosen actuation technology, the designer can then be able to fix the main geometrical features of the instrument.

Although the presented method allows the calculation of specific design-oriented metrics for the design of steerable flexible instrument for maxillary sinus surgery, some important aspects should be taken into account when interpreting the results. First, it should be noted that the used sample size (20 CT scans, giving 20 left-side and 20 right-side surface meshes of the maxillary sinus 
and the nasal cavity) is limited. In order to cover more variations in sinuses, a more extensive data set would be needed for future study. The key contribution of this study is, however, the workflow used to deduce design-oriented metrics based on a SSM of the maxillary sinus and the nasal cavity. Current results can already be used for the design of first prototypes. Second, the equation used to calculate the percentage of the subject set that has a nasal cavity width larger than a specific arbitrary limit (Eq. (5)) underestimates this percentage. This is due to the fact that when one of both variations $\pm 3 \sqrt{\lambda_{t}}$ is exceeding the width arbitrary limit, the entire half side of the Gaussian curve is discarded. All the variations within $\pm 3 \sqrt{\lambda_{t}}$ were not tested. Finally, the derived design-oriented metrics could be used as a good reference in the design phase. It would be recommended, however, to verify the results experimentally e.g. on an in-silico model or on cadavers, which provide a more realistic rendering of the nasal cavity and sinuses environment.It is also important to note that the presented method can also be applied to other clinical applications featuring complex and confined 3D anatomical spaces, including lumina and cavities, like frontal sinus surgery, olfactory cleft inspection or interventional cardiology.

\section{Acknowledgments}

This work was supported by a grant from the Belgian FWO [SB/1S98418N]. 


\section{References}

1. Abd-alla, M. A. and A.-J. J. Mahdi. Maxillary sinus measurements in different age groups of human cadavers. Tikrit Journal for Dental Sciences 3:107-112, 2014.

2. Albrecht, T., M. Luthi, and T. Vetter. A statistical deformation prior for non-rigid image and shape registration. In: 2008 IEEE Conference on Computer Vision and Pattern Recognition, pp. 1-8. 2008.

3. Ariji, Y., E. Ariji, K. Yoshiura, and S. Kanda. Computed tomographic indices for maxillary sinus size in comparison with the sinus volume. Dentomaxillofacial Radiology 25:19-24, 1996.

4. Chee, L. and D. Sethi. The endoscopic management of sinonasal inverted papillomas 1. Clinical Otolaryngology \& Allied Sciences 24:61-66, 1999.

5. Cootes, T. F., C. J. Taylor, D. H. Cooper, and J. Graham. Active shape models-their training and application. Computer vision and image understanding 61:38-59, 1995.

6. Danckaers, F., T. Huysmans, D. Lacko, A. Ledda, S. Verwulgent, S. Van Dongen, and J. Sijbers. Correspondence preserving elastic surface registration with shape model prior. In: 2014 22nd International Conference on Pattern Recognition, pp. 2143-2148. 2014.

7. Dkhar, W., A. Pradhan, and M. Shajan. Measurement of different dimension of maxillary and frontal sinus through computed tomography. Online J Health Allied Scs 16:5, 2017. 
8. Giacomini, G., A. L. M. Pavan, J. M. C. Altemani, S. B. Duarte, C. M. C. B. Fortaleza, J. R. de Arruda Miranda, and D. R. De Pina. Computed tomography-based volumetric tool for standardized measurement of the maxillary sinus. PloS one 13:e0190770, 2018.

9. Gosau, M., D. Rink, O. Driemel, and F. Draenert. Maxillary sinus anatomy: a cadaveric study with clinical implications. The Anatomical Record: Advances in Integrative Anatomy and Evolutionary Biology: Advances in Integrative Anatomy and Evolutionary Biology 292:352$354,2009$.

10. Heimann, T. and H.-P. Meinzer. Statistical shape models for 3d medical image segmentation: a review. Medical image analysis 13:543-563, 2009.

11. Hong, W., L. Xie, J. Liu, Y. Sun, K. Li, and H. Wang. Development of a novel continuum robotic system for maxillary sinus surgery. IEEE/ASME Transactions on Mechatronics 23:1226-1237, 2018.

12. Iwanaga, J., C. Wilson, S. Lachkar, K. A. Tomaszewski, J. A. Walocha, and R. S. Tubbs. Clinical anatomy of the maxillary sinus: application to sinus floor augmentation. Anatomy \& cell biology 52:17-24, 2019.

13. Jun, B.-C., S.-W. Song, C.-S. Park, D.-H. Lee, K.-J. Cho, and J.-H. Cho. The analysis of maxillary sinus aeration according to aging process; volume assessment by 3-dimensional reconstruction by high-resolutional ct scanning. Otolaryngology-Head and Neck Surgery 132:429-434, 2005. 
14. Kamel, R. H. Conservative endoscopic surgery in inverted papilloma: preliminary report. Archives of Otolaryngology-Head \& Neck Surgery 118:649-653, 1992.

15. Kenned, D. W., S. J. Zinreich, F. Kuhn, H. Shaalan, R. Naclerio, and E. Loch. Endoscopic middle meatal antrostomy: theory, technique, and patency. The Laryngoscope 97:1-9, 1987.

16. Keustermans, W., T. Huysmans, B. Schmelzer, J. Sijbers, and J. J. Dirckx. Matlab® toolbox for semi-automatic segmentation of the human nasal cavity based on active shape modeling. Computers in biology and medicine 105:27-38, 2019.

17. Kiruba, L. N., C. Gupta, S. Kumar, A. S. D'Souza et al. A study of morphometric evaluation of the maxillary sinuses in normal subjects using computer tomography images. Archives of Medicine and Health Sciences 2:12, 2014.

18. Kozic, N., S. Weber, P. Büchler, C. Lutz, N. Reimers, M. Á. G. Ballester, and M. Reyes. Optimisation of orthopaedic implant design using statistical shape space analysis based on level sets. Medical image analysis 14:265-275, 2010.

19. Legrand, J., D. Dirckx, M. Durt, M. Ourak, J. Deprest, S. Ourselin, J. Qian, T. Vercauteren, and E. Vander Poorten. Active handheld flexible fetoscope-design and control based on a modified generalized prandtl-ishlinski model. IEEE/ASME International Conference on Advanced Intelligent Mechatronics, 2020.

20. Sadeghi, N., S. Al-Dhahri, and J. J. Manoukian. Transnasal endoscopic medial maxillectomy for inverting papilloma. The Laryngoscope 113:749-753, 2003. 
21. Sahlstrand-Johnson, P., M. Jannert, A. Strömbeck, and K. Abul-Kasim. Computed tomography measurements of different dimensions of maxillary and frontal sinuses. BMC medical imaging 11:8, 2011.

22. Sarkalkan, N., H. Weinans, and A. A. Zadpoor. Statistical shape and appearance models of bones. Bone 60:129-140, 2014.

23. Schneider, J. S., J. Burgner, R. J. Webster III, and P. T. Russell III. Robotic surgery for the sinuses and skull base: What are the possibilities and what are the obstacles? Current opinion in otolaryngology \& head and neck surgery 21:11, 2013.

24. Sharma, S. K., M. Jehan, and A. Kumar. Measurements of maxillary sinus volume and dimensions by computed tomography scan for gender determination. Journal of the anatomical society of India 63:36-42, 2014.

25. Sinha, A., S. Leonard, A. Reiter, M. Ishii, R. H. Taylor, and G. D. Hager. Automatic segmentation and statistical shape modeling of the paranasal sinuses to estimate natural variations. Medical Imaging 2016: Image Processing 9784:97840D, 2016.

26. Souza, A. D., K. Rajagopal, V. H. Ankolekar, A. S. D. Souza, S. R. Kotian et al. Anatomy of maxillary sinus and its ostium: A radiological study using computed tomography. CHRISMED Journal of Health and Research 3:37, 2016.

27. Storz, K. Karl storz - endoscopes and instruments for ent esophagoscopy - bronchoscopy. https://www.karlstorz.com/cps/rde/xbcr/karlstorz_assets/ASSETS/3331528.pdf (Accessed date: 16/03/2020), 2020. 
28. Tichenor, W. S., A. Adinoff, B. Smart, and D. L. Hamilos. Nasal and sinus endoscopy for medical management of resistant rhinosinusitis, including postsurgical patients. Journal of allergy and clinical immunology 121:917-927, 2008.

29. Uchida, Y., M. Goto, T. Katsuki, and T. Akiyoshi. A cadaveric study of maxillary sinus size as an aid in bone grafting of the maxillary sinus floor. Journal of oral and maxillofacial surgery 56:1158-1163, 1998.

30. Vrancken, A., S. Crijns, M. Ploegmakers, C. O’kane, T. G. van Tienen, D. Janssen, P. Buma, and N. Verdonschot. 3d geometry analysis of the medial meniscus-a statistical shape modeling approach. Journal of anatomy 225:395-402, 2014.

31. Wormald, P. J., E. Ooi, C. A. van Hasselt, and S. Nair. Endoscopic removal of sinonasal inverted papilloma including endoscopic medial maxillectomy. The Laryngoscope 113:867$873,2003$.

32. Yoon, H.-S., J. H. Jeong, and B.-J. Yi. Image-guided dual master-slave robotic system for maxillary sinus surgery. IEEE Transactions on Robotics 34:1098-1111, 2018. 


\section{Appendix A: Extracted landmarks}
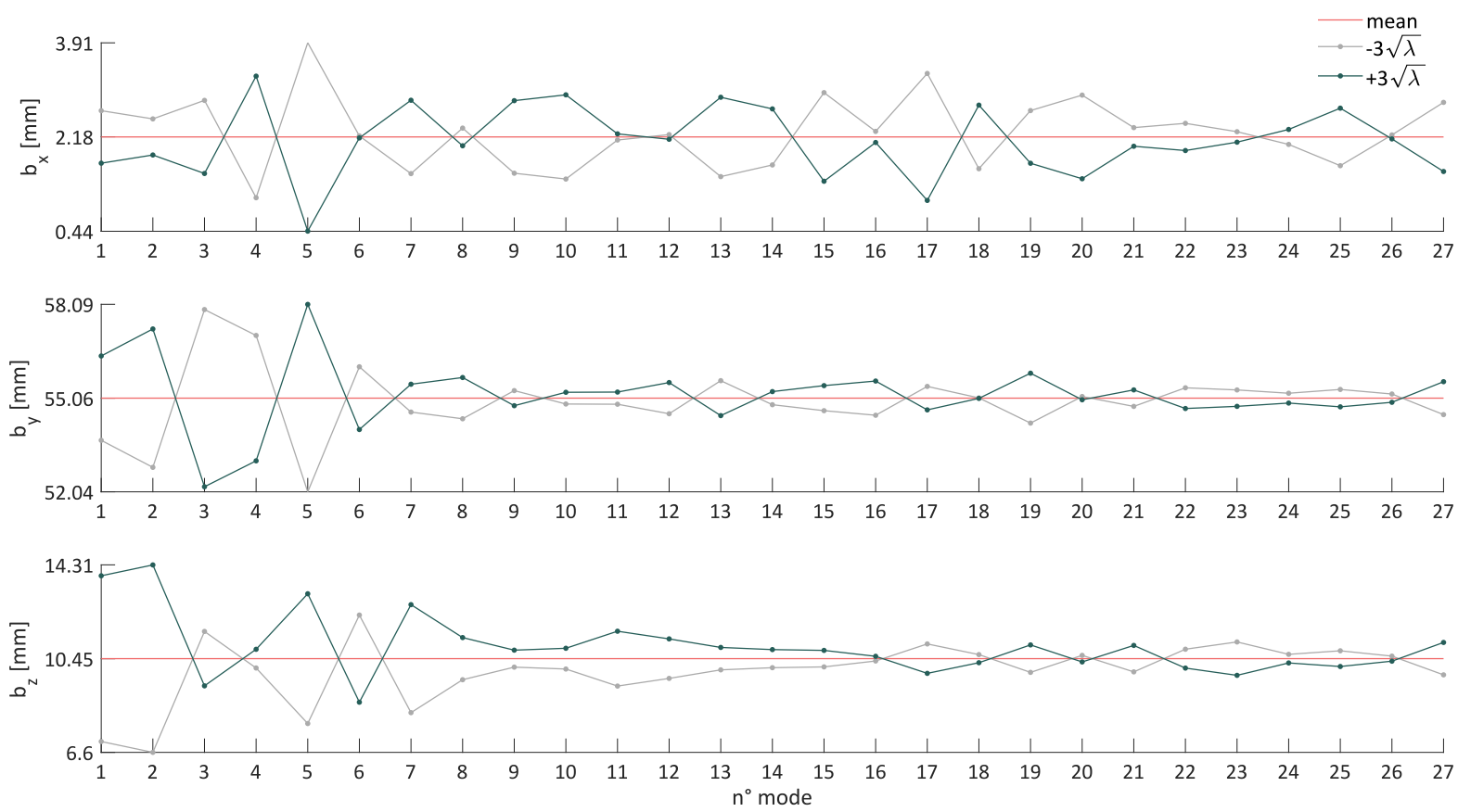

Figure 9: Extracted antrostomy window center point landmark $b$ values from the mean shape and the $\pm 3 \sqrt{\lambda_{t}}$ variations of the 27 first shape modes in [mm]. 

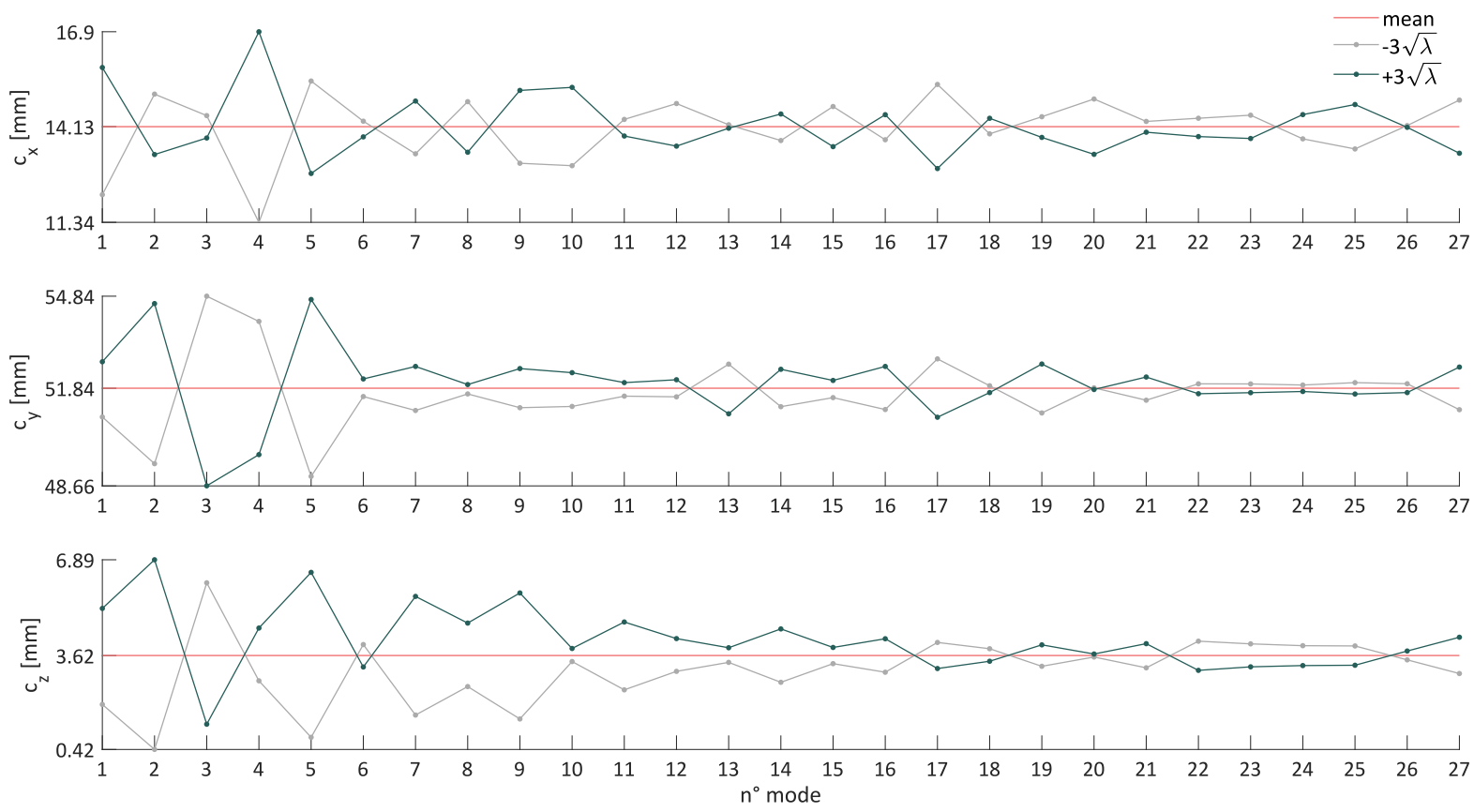

Figure 10: Extracted maxillary sinus center point landmark $c$ values from the mean shape and the $\pm 3 \sqrt{\lambda_{t}}$ variations of the 27 first shape modes in [mm].
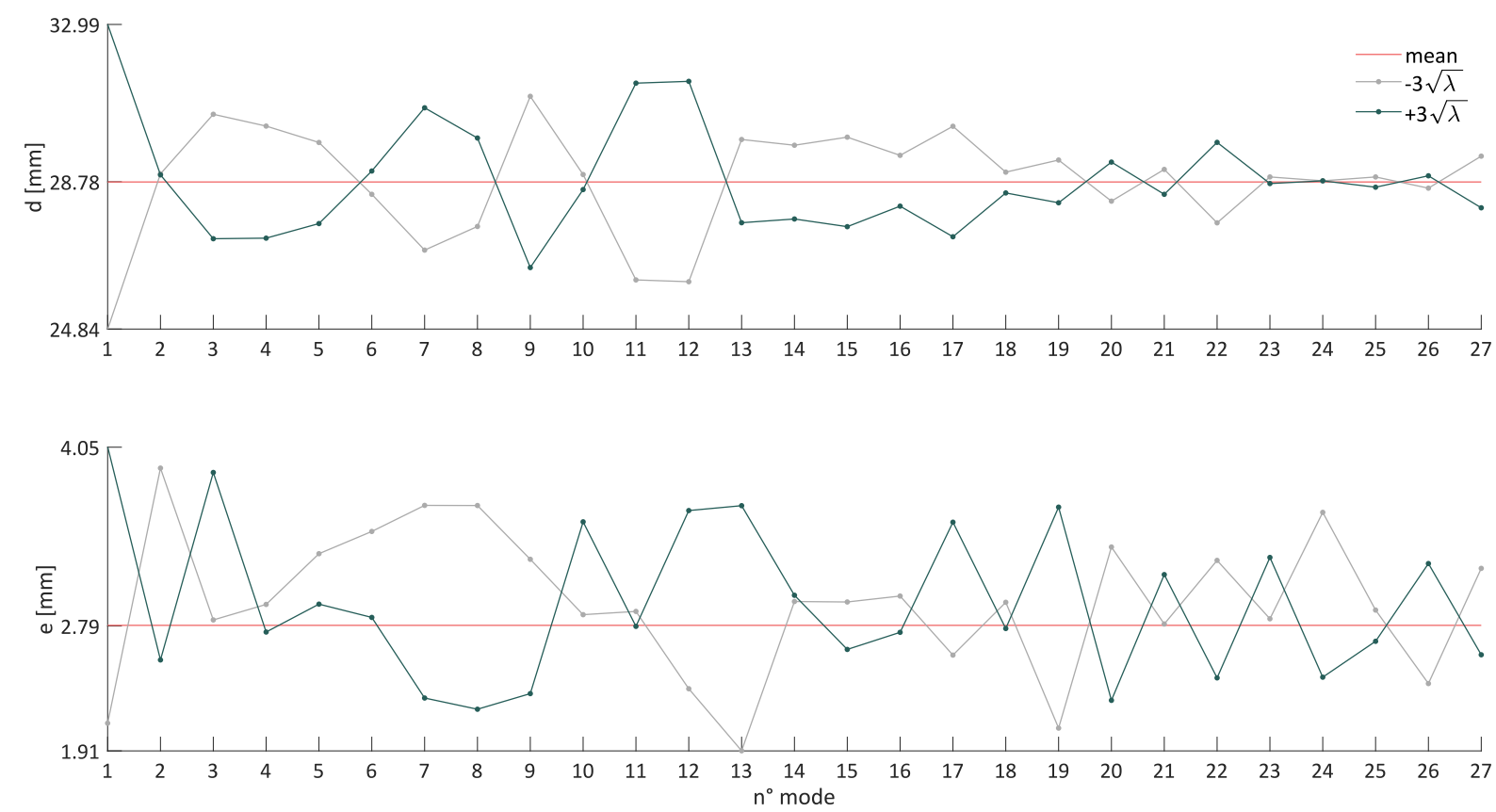

Figure 11: Extracted antrostomy window length $d$ thickness and $e$ landmarks values from the mean shape and the $\pm 3 \sqrt{\lambda_{t}}$ variations of the 27 first shape modes in [mm]. 

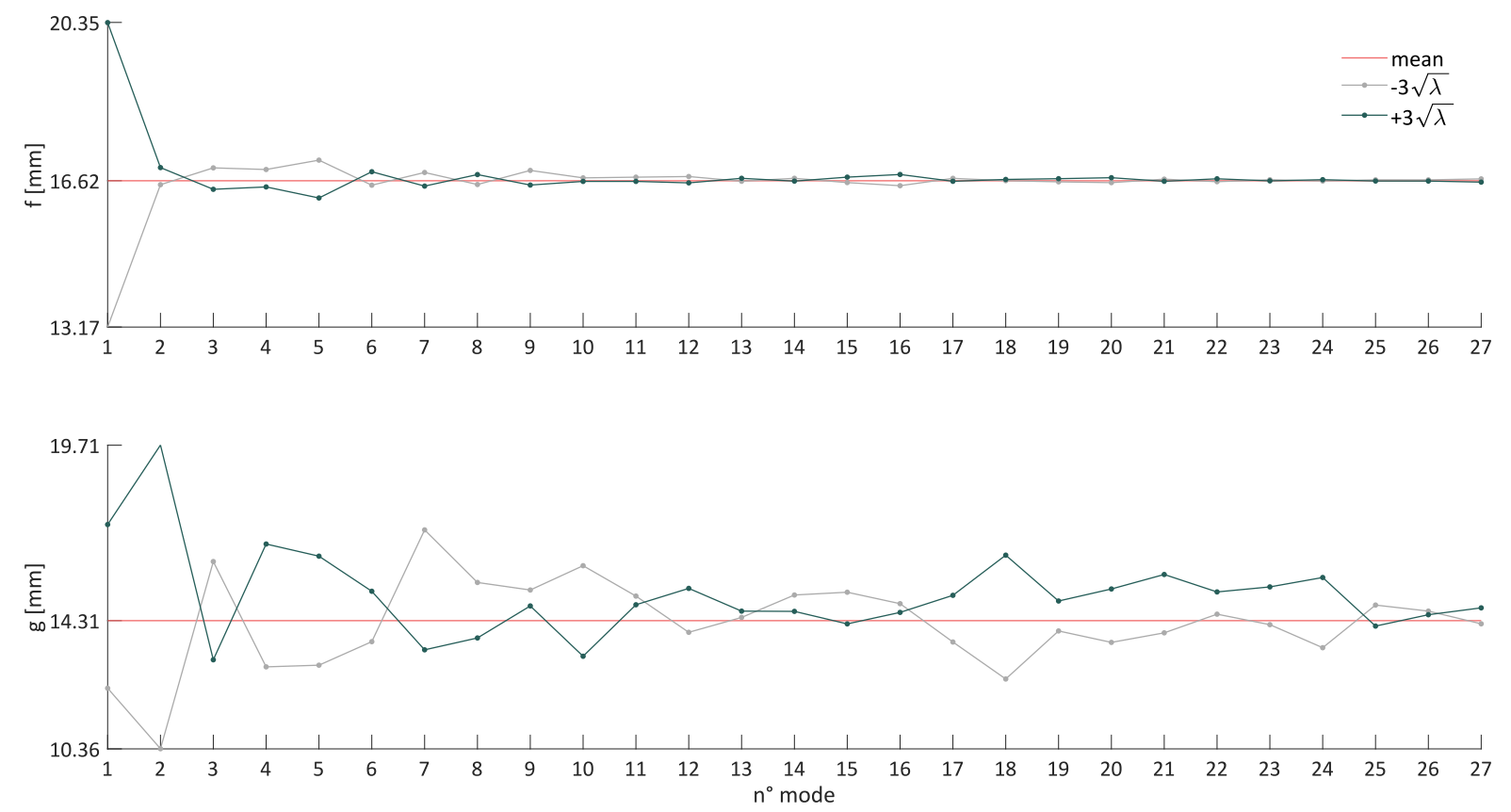

Figure 12: Extracted radius of the maxillary sinus fitting sphere $f$ and antrostomy window width $g$ landmarks values from the mean shape and the $\pm 3 \sqrt{\lambda_{t}}$ variations of the 27 first shape modes in [mm].
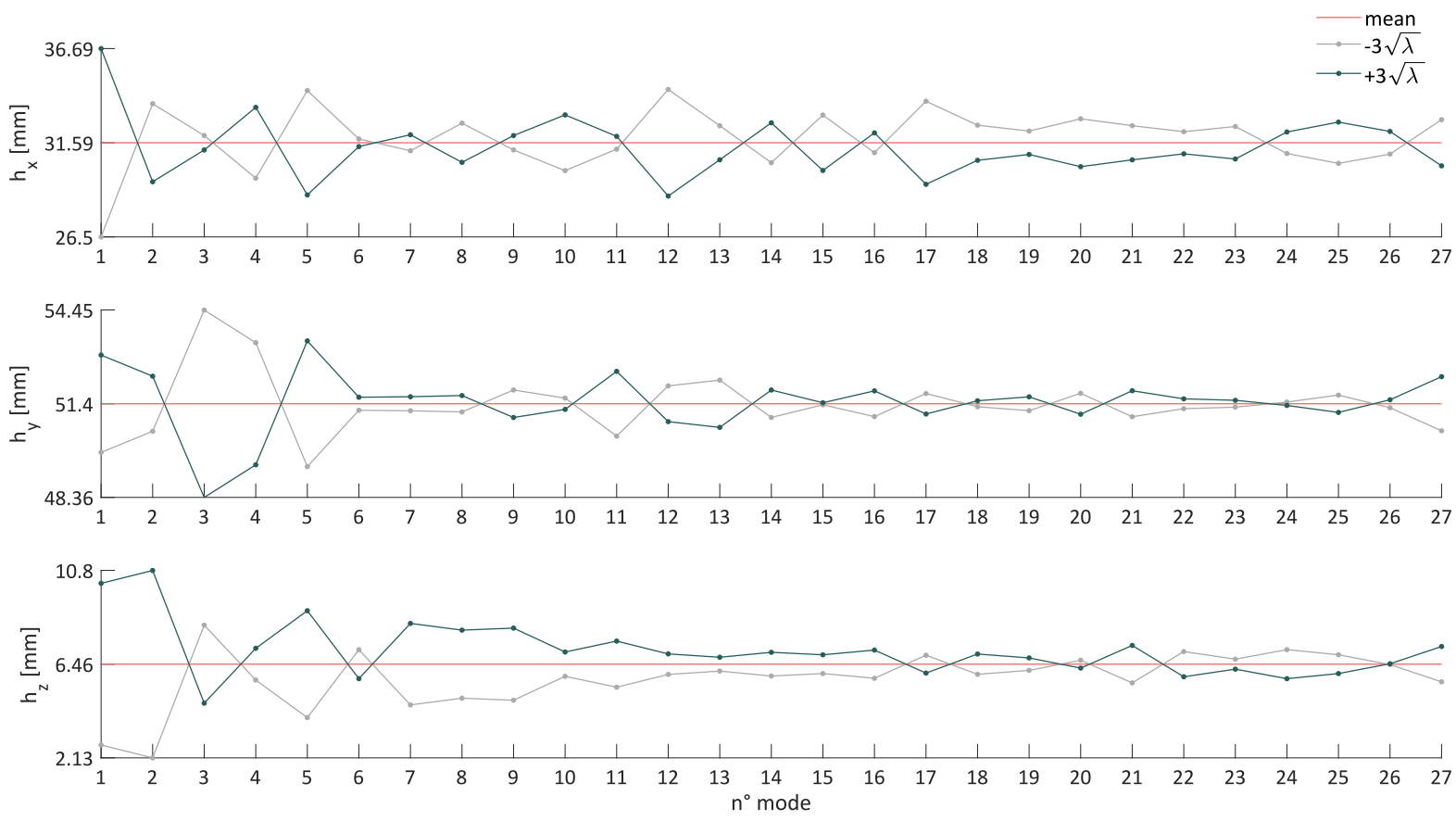

Figure 13: Extracted most lateral point of the maxillary sinus landmark $h$ values from the mean shape and the $\pm 3 \sqrt{\lambda_{t}}$ variations of the 27 first shape modes in [mm]. 

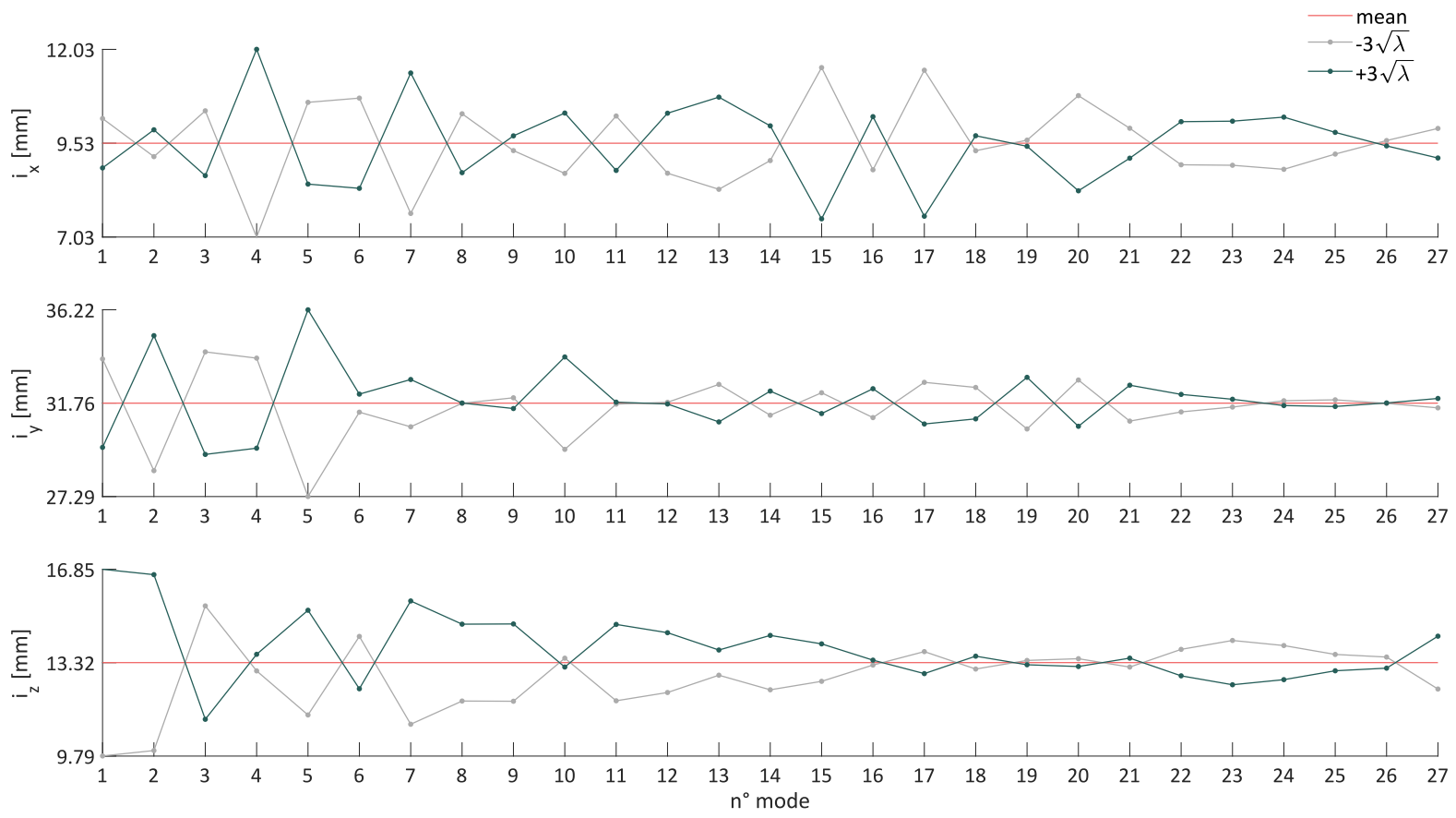

Figure 14: Extracted most anterior point of the maxillary sinus landmark $i$ values from the mean shape and the $\pm 3 \sqrt{\lambda_{t}}$ variations of the 27 first shape modes in [mm]. 